\title{
High-Precision Size Recognition and Separation in Synthetic 1D Nanochannels
}

\author{
Ping Wang, Xinyi Chen, Qiuhong Jiang, Matthew Addicoat, Ning Huang, Sasanka Dalapati, \\ Thomas Heine, Fengwei Huo, and Donglin Jiang*
}

\begin{abstract}
Covalent organic frameworks (COFs) allow elaborate manufacture of ordered one-dimensional channels in the crystal. We defined a superlattice of COFs by engineering channels with a persistent triangular shape and discrete pore size. We observed a size-recognition regime that is different from the characteristic adsorption of COFs, whereby pore windows and walls were cooperative so that triangular apertures sorted molecules of one-atom difference and notch nanogrooves confined them into single-file molecular chains. The recognition and confinement were accurately described by sensitive spectroscopy and femtosecond dynamic simulations. The resulting COFs enabled instantaneous separation of mixtures at ambient temperature and pressure. This study offers an approach to merge precise recognition, selective transport, and instant separation in synthetic $1 D$ channels.
\end{abstract}

\section{Introduction}

One-dimensional (1D) nanochannels offer an ideal molecular conduit for mass transport, as observed in biological ion channels and synthetic porous materials. ${ }^{[1]}$ Assimilating a molecular-size-recognition regime into $1 \mathrm{D}$ channels could create materials with exceptional separation and selective transportation properties. Besides accurate pore dimensions and shape, ion channels enable size recognition by precise

[*] Dr. P. Wang, X. Chen, Dr. Q. Jiang, Dr. N. Huang, Dr. S. Dalapati, Prof. Dr. D. Jiang

Department of Chemistry, Faculty of Science

National University of Singapore

3 Science Drive 3, Singapore 117543 (Singapore)

E-mail:chmjd@nus.edu.sg

X. Chen, Prof. Dr. F. Huo

Key Laboratory of Flexible Electronics and Institute of Advanced Materials, Nanjing Tech University

30 South Puzhu Road, Nanjing 211816 (P. R. China)

Dr. M. Addicoat

School of Science and Technology, Nottingham Trent University

Nottingham, NG11 8NS (UK)

Prof. Dr. T. Heine

Department of Theoretical Chemistry, TU Dresden

Mommsenstrasse 13, 01062 Dresden (Germany)

Prof. Dr. D. Jiang

Joint School of National University of Singapore and Tianjin

University, International Campus of Tianjin University

Binhai New City, Fuzhou 350207 (P. R. China)

(2) Supporting information and the ORCID identification number(s) for

the author(s) of this article can be found under:

https://doi.org/10.1002/anie.201909851. spatial organization of polypeptide segments on the pore wall as a checkpoint to identify ions. ${ }^{[1 \mathrm{a}]}$ Artificial 1D nanochannels can be prepared using three major methods, including phaseseparation-driven self-assembly of block copolymers to form a nanodomain structure followed by chemical etching to yield cylindrical nanoholes, ${ }^{[2]}$ liquid-crystal-templated silication to form mesoporous hexagonal silicates, ${ }^{[3]}$ and topology-directed polycondensation to produce covalent organic frameworks (COFs) with built-in polygonal pores. ${ }^{[4]}$ However, these straight channels usually have uniform chemical compositions of the pore wall, thus precluding the possibility of manufacturing specific wall sectors to create a checkpoint for size recognition. Thus, engineering synthetic $1 \mathrm{D}$ channels to develop size-recognition properties is a challenge.

For the synthesis of covalent organic frameworks (COFs), various organic units are elaborated to generate extended crystalline frameworks through polycondensation, whereby covalent-bond and noncovalent interactions are integrated to direct the covalent growth of two-dimensional (2D) polymer sheets and to control the mode of $\pi$ stacking to construct ordered yet dense 1D synthetic nanochannels across the material. ${ }^{[5]}$ Owing to the broad diversity of building units, COFs contain 1D channels with different polygon shapes ranging from hexagonal to tetragonal and trigonal. These 1D channels have been developed for the adsorption and transport of various molecules. ${ }^{[1,5]}$ However, use of the 1D channels of COFs for size recognition has not been well explored.

Herein we show a strategy for developing 1D channels of COFs to recognize molecular size. We observed a sizerecognition mechanism that is different from the typical adsorption behavior of COFs, whereby the pore windows and walls are cooperative so that the shape-persistent triangular apertures can sort molecules of one-atom difference, and the notch nanogrooves can confine them into single-file molecular chains. Moreover, we scrutinized the structural origins of the size-recognition regime in the synthetic 1D channels and demonstrate their exceptional functions in size recognition and separation. We elucidated the structure-function correlation and disclose the structural parameters that control the size-recognition process. We highlight the ability of the frameworks to combine precise recognition, selective transport, and instant separation in one material. 


\section{Results and Discussion}

We designed and synthesized artificial 1D channels that enable accurate size recognition by topology-directed lattice engineering of COFs (Figure 1, HFPTP-BPDA-COF). We developed a superlattice of $1 \mathrm{D}$ channels with a persistent triangular shape and discrete pore size. The HFPTP-BPDACOF was synthesized by topology-guided polycondensation of $C_{3}$-symmetric 2,3,6,7,10,11-hexakis(4-formylphenyl)triphenylene (HFPTP) and $C_{2}$-symmetric 1,1'-biphenyl-4,4'-diamine (BPDA) under solvothermal conditions (Figure $1 \mathrm{a}$; see also Figure S1 in the Supporting Information).

Each channel consists of three $\mathrm{V}$-shaped nanogrooves at three corners (Figure $1 \mathrm{~b}, \mathrm{c}$ ) and is formed by $\pi$ stacking of a triangular macrocycle (Figure $1 \mathrm{~b}$ ); the three equivalent walls are covered by sequenced zigzag $\mathrm{C}-\mathrm{H}$ units extruded from the backbones (Figure 1c) and readily accessible to guest molecules. The triangular superlattice is covalently fixed by the two-dimensional (2D) sheet (Figure 1d) and further locked by $\pi$ stacking (Figure $1 \mathrm{e}$ ). We unexpectedly found that the triangular channels enable size recognition to identify molecules of one-atom difference, thus opening an unprecedented strategy that can integrate precise recognition, selective transport, and instant separation into one channel.

The chemical structure of HFPTP-BPDA-COF was characterized by various analytical methods (see Figures S2-S4 and Tables S1-S3 in the Supporting Information). Fourier transform infrared spectroscopy (see Figure S2) revealed a new peak at $1620 \mathrm{~cm}^{-1}$ assignable to the imine linkage, ${ }^{[6]}$ thus indicating the polycondensation between HFPTP and BPDA (see Figure S3). Solid-state ${ }^{13} \mathrm{C}$ NMR spectroscopy (see Figure S3) revealed that a peak at $157 \mathrm{ppm}$ that could be assigned to the $\mathrm{C}$ atom of the $\mathrm{C}=\mathrm{N}$ bond, thus indicating the formation of imine linkages in HFPTP-BPDACOF $^{[6]}$ Thermogravimetric analysis suggested that HFPTPBPDA-COF was stable up to $500^{\circ} \mathrm{C}$ under nitrogen (see Figure S4).

HFPTP-BPDA-COF exhibited powder X-ray diffraction (PXRD) signals at $3.42^{\circ}, 5.98^{\circ}, 6.96^{\circ}, 9.26^{\circ}, 10.38^{\circ}$, and $24.71^{\circ}$, which were assigned to the (100), (110), (200), (210), (300), and (001) facets, respectively (Figure $2 \mathrm{a}$, red curve). The presence of the (001) facet suggests structural ordering with $3.64 \AA$ separation in the $z$ direction perpendicular to the $2 \mathrm{D}$ layers (Figure 1e). We used density functional based tight binding $(\mathrm{DFTB}+)$ calculations $^{[7]}$ to optimize the conformation of the 2D single layer and the configurations of different stacking modes (see Table S1). The energetically most favorable slipped AA stacking mode yielded a PXRD pattern (Figure 2a, blue curve) that is in good agreement with the experimentally observed profile. Pawley refinement using the P1 space group (Figure $2 \mathrm{a}$, green curve) with the unit-cell parameters of $a=30.0679 \AA, b=30.0863 \AA, c=7.6352 \AA, \alpha=$ $89.8782^{\circ}, \beta=90.1544^{\circ}$, and $\gamma=60.0872^{\circ}$ reproduced the PXRD pattern with negligible deviation (Figure $2 \mathrm{a}$, black curve; see Tables S2 and S3 for a summary of the atomic coordinates of DFTB + and Pawley refinement). Thus, the reconstruction reveals an extended dual-pore superlattice with ordered triangular 1D channels (Figure 1d).
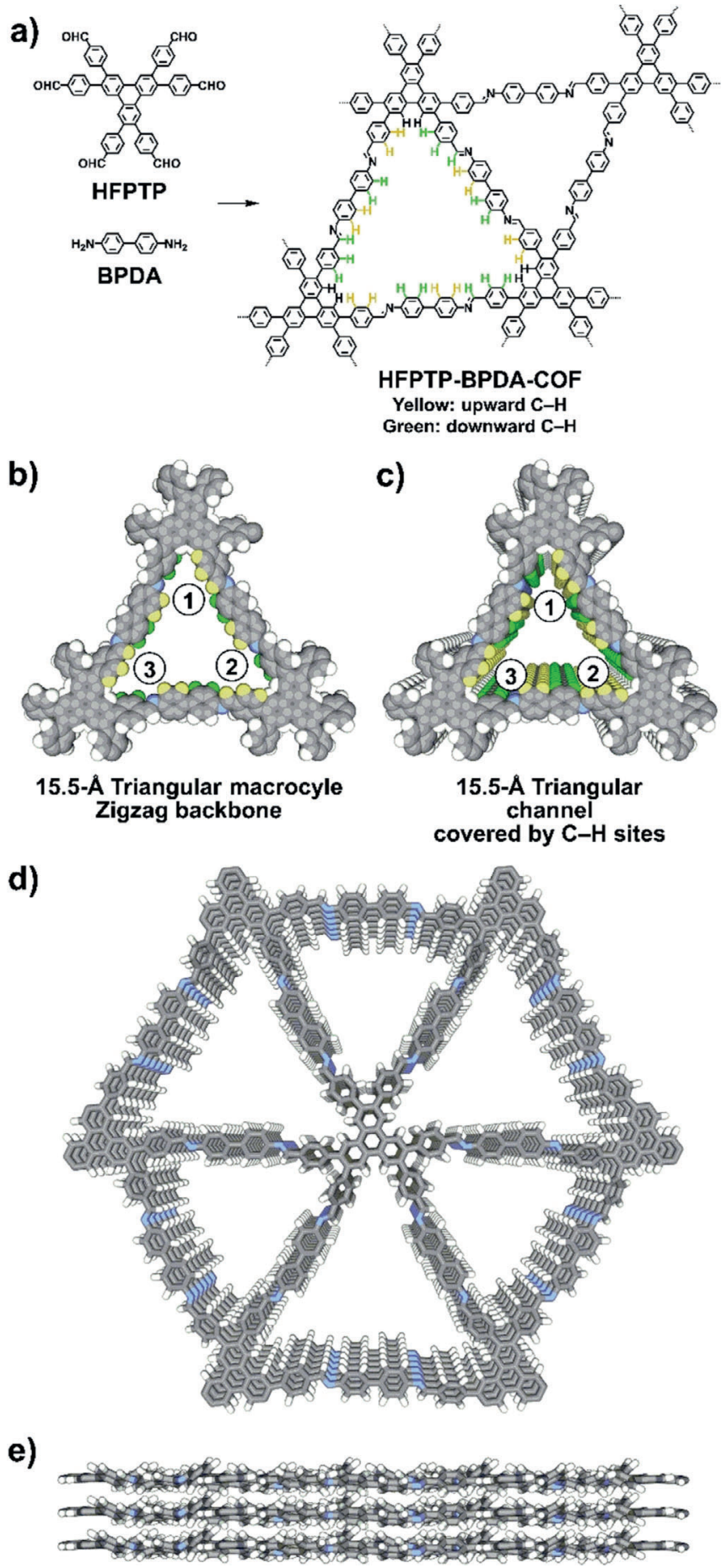

Figure 1. a) Synthesis of HFPTP-BPDA-COF. b, c) Reconstructed crystal structures of one triangular macrocycle with zigzag backbones and a $15.5 \AA$ pore size (b) and many layers of the 1D $15.5 \AA$ triangular channel covered by sequenced upward and downward $\mathrm{C}-\mathrm{H}$ sites (c; yellow for upward $\mathrm{C}-\mathrm{H}$ and green for downward $\mathrm{C}-\mathrm{H}$ sites relative to the $2 \mathrm{D}$ sheet). Each triangular channel consists of three equivalent $\mathrm{V}$ shaped corner nanogrooves (indicated 1, 2, and 3) with readily accessible $\mathrm{C}-\mathrm{H}$ sites. d) Top and e) side view of the reconstructed crystal structure of the extended dual-pore triangular superlattice. 
a)

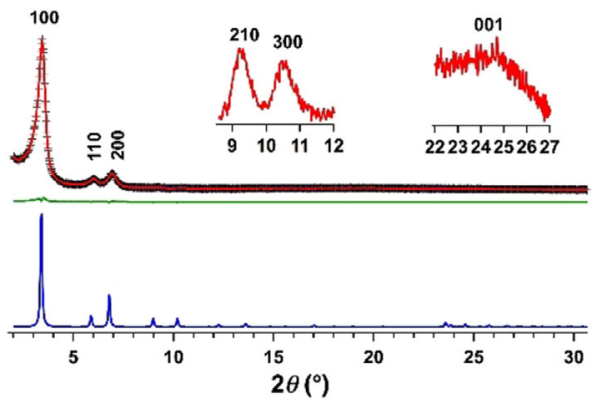

b)

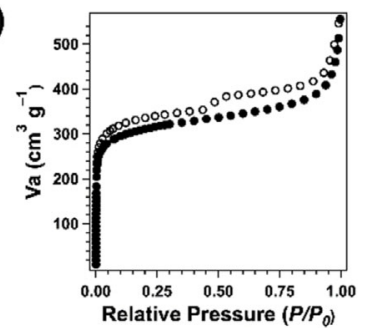

c)

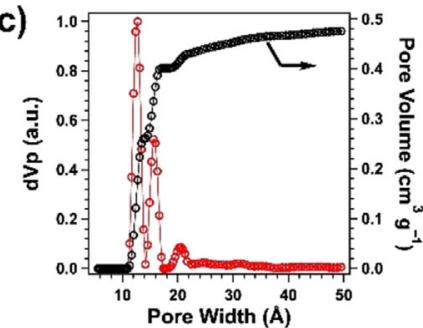

Figure 2. a) PXRD profiles of HFPTP-BPDA-COF: Experimentally observed PXRD pattern (red curve), Pawley-refined PXRD pattern (black curve), the difference between them (green curve), and simulated pattern with the $2.0 \AA$ slipped AA stacking model (blue curve). The insets are the enlarged PXRD patterns. b) Nitrogen-sorption isotherm curves measured at $77 \mathrm{~K}$ (filled circles for sorption and open circles for desorption). c) Profiles of the pore-size distribution (red dots) and pore-volume distribution (black dots).

HFPTP-BPDA-COF exhibited reversible nitrogen-sorption isotherm curves (Figure $2 \mathrm{~b}$ ) with a Brunauer-EmmettTeller (BET) surface area of $1024 \mathrm{~m}^{2} \mathrm{~g}^{-1}$. The pore-sizedistribution profile revealed the presence of two different pores with a size of 12.7 and $15.5 \AA$, respectively (Figure $2 \mathrm{c}$ ), which is consistent with the superlattice structure (Figure 1d). The $2.1 \mathrm{~nm}$ mesopore is attributed to a structural defect caused by a missed linkage between neighboring pores, which endows the isotherm curves with a hysteresis loop. As the $15.5 \AA$ trigonal channels and mesopores are independent without any interpenetration between them, the presence of mesopore defects does not exert any influence on the recognition behavior of COFs. As the mesopore has a pore volume of $0.03 \mathrm{~cm}^{3} \mathrm{~g}^{-1}$, the percentage of mesopore defects is low.

We probed the size recognition of HFPTP-BPDA-COF using electronic absorption spectroscopy. Nile red (NR), 7 (diethylamino)-3-phenylcoumarin (DAPC), and Coumarin 6 (C6) (Figure $3 \mathrm{a}$ ) are similar in their backbones and are bulky (contain 40 or more atoms). There is a difference of one atom between NR and C6. The big difference in the wavelengths of the absorption bands of NR $(523 \mathrm{~nm})$, DAPC (393 nm), and C6 $(446 \mathrm{~nm})$ allows for the distinct detection of each compound in a mixture; their high absorption coefficients $\left(>36000 \mathrm{M}^{-1}\right)$ enable quantitative and sensitive monitoring of the recognition process.

We added the HFPTP-BPDA-COF crystallites to a THF solution of NR (15.0 ̊ in size) and monitored the resulting solution by time-dependent spectroscopy. After only $1 \mathrm{~min}$, a sharp drop in the NR absorbance was observed (Figure 3b), indicating the rapid uptake of NR by HFPTP-BPDA-COF.

a)
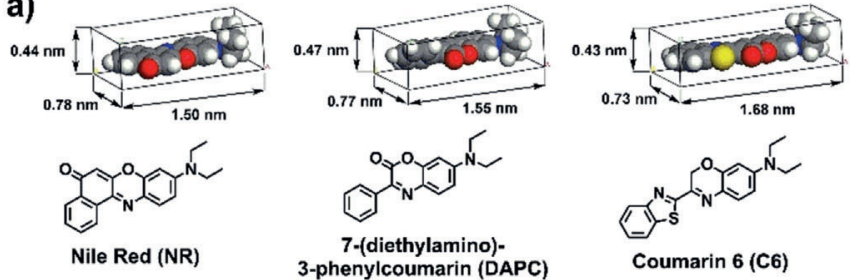

b)
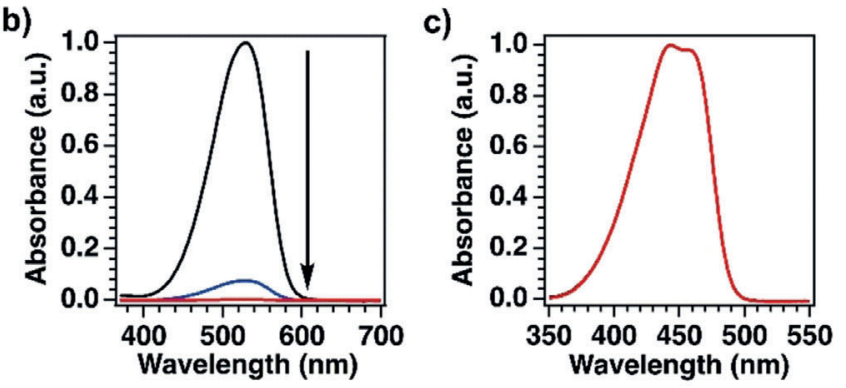

d)
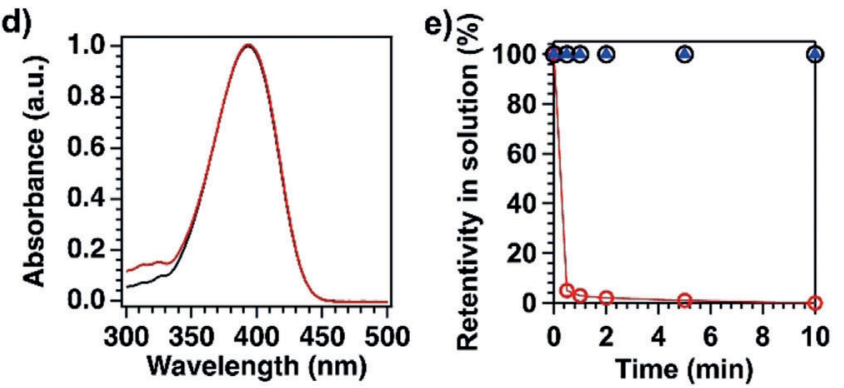

Figure 3. a) Chemical structure and dimensions of Nile red (NR), 7(diethylamino)-3-phenylcoumarin (DAPC), and Coumarin 6 (C6). b) Electronic absorption spectral change of a solution of NR in THF upon addition of the insoluble HFPTP-BPDA-COF crystallites (black curve, pristine solution; blue curve, 1 min after addition; red curve, 2 min after addition). c, d) Electronic absorption spectra of solutions of C6 (c) and DAPC (d) in THF upon the addition of HFPTP-BPDA-COF (black curve, pristine solution; red curve, $12 \mathrm{~h}$ after addition; overlapped). e) Time-dependent retentivity of NR (red circles and line), C6 (blue triangles), and DAPC (black circles) in the THF solutions after the addition of HTP-BDA-COF.

Notably, HFPTP-BPDA-COF took up nearly all NR molecules within 2 min (Figure $3 \mathrm{~b}$, red curve; Figure $3 \mathrm{e}$, red circles). In contrast, under otherwise identical conditions, a solution of C6 (16.8 $\mathrm{A}$ in size) in THF did not show any spectral change even after $12 \mathrm{~h}$ (Figure $3 \mathrm{c}$; Figure $3 \mathrm{e}$, blue triangles). This result suggests that the triangular channels completely prevent the entry of $\mathrm{C} 6$, which is larger than NR by one atom. Furthermore, DAPC (15.5 $\AA$ ), which is comparable in size to the pore aperture $(15.5 \AA)$, was also rejected by the channels, and showed no spectral change upon the addition of HFPTP-BPDA-COF, irrespective of immersion time (Figure 3d; Figure 3e, black circles). These clear differences revealed that the triangular aperture in HFPTP-BPDACOF serves as a checkpoint to recognize a subtle difference in size and to sort molecules into 1D channels.

To verify that the uptake occurred in the channels, we investigated the nitrogen-sorption isotherms of HFPTPBPDA-COF after NR uptake. The resulting COF samples exhibited a sharp decrease in the BET surface area from 1024 to $730 \mathrm{~m}^{2} \mathrm{~g}^{-1}$ (see Figure S5a). More specifically, the pore- 
size-distribution profile (see Figure $\mathrm{S} 5 \mathrm{~b}$ ) revealed that the $15.5 \AA$ channel almost disappeared. The pore-volume profile (see Figure S5c) revealed that the $12.7 \AA$ pore did not show any change and the $15.5 \AA$ channels were fully occupied. As a control, HFPTP-BPDA-COF after addition to the DAPC solution did not change the nitrogen-sorption profiles, as DAPC cannot enter the pores (see Figure S6). Therefore, the 15.5 A channels account for the recognition and nanoconfinement. Furthermore, the $2.1 \mathrm{~nm}$ mesopore was well-retained without any change in the pore volume of $0.03 \mathrm{~cm}^{3} \mathrm{~g}^{-1}$, thus demonstrating that the mesopore is not involved in guest uptake.

To confirm whether the recognition was dependent on the channel shape, we conducted control experiments using tetragonal and hexagonal $\mathrm{COFs}^{[6,8]}$ that have the same pore size as that of HFPTP-BPDA-COF. We observed that neither hexagonal TTA-TFB-COF (see Figure S7a-e) nor tetragonal TFBCz-PDA-COF (see Figure S7 f-j) caused a change in the absorption spectrum of NR even after $12 \mathrm{~h}$. These results rationalize our strategy by confirming that the triangular shape is of fundamental importance for size recognition.

A possible scenario of the process was revealed by molecular dynamic simulations at the femtosecond level using a global minimum energy protocol. $^{[9]}$ The guest molecules approaching HFPTP-BPDA-COF adopt an orientation with their $\pi$ backbone parallel to the COF $\pi$ surface (the (100) facet), whereby the potential energy is minimized (Figure $4 \mathrm{a}$, stage 1). If guest molecules, such as DAPC and $\mathrm{C} 6$, are larger than the window, the triangular aperture blocks their entry (see Figure S8) so that they are left in solution. Once the NR molecules pass the aperture (Figure 4a, stage 2), they rotate in the channels to take a diving pose (Figure $4 \mathrm{a}$, stage 3 ) with their $\pi$ backbones parallel to the long axis of channels and are successively confined at the corner nanogrooves (Figure $4 \mathrm{a}$, stage 4 ) to form single-file molecular chains in the channel (Figure 4d). These four processes are complete within 10 ps. Each $15.5 \AA$ sized channel has three nanogrooves and confines three single-file molecular chains (Figure 4b,c); an $18 \mathrm{~nm}$ long channel can dock $30 \mathrm{NR}$ molecules into 3 single-file chains at the nanogrooves (Figure $4 \mathrm{~d}$ ). From the density of the $15.5 \AA$ channels, a cubic HFPTP-BPDA-COF crystallite with a dimension of only $500 \times 500 \times 500 \mathrm{~nm}^{3}$ confines as many as 7500000 NR molecules (Figure 4d). This exceptional confinement originates from the full accessibility of every channel corner in the crystal. Kinetics experiments (see Figure S9) revealed an apparent pseudo-second-order rate constant $\left(k_{\text {obs }}\right)$ of $13.11 \mathrm{mg} \mathrm{g}^{-1} \mathrm{~min}^{-1}$, which is more than three orders of magnitude higher than those of the state-of-the-art $\mathrm{COFs}^{[6]}$ and amorphous porous polymers. ${ }^{[10]}$ The high $k_{\mathrm{obs}}$ value also suggests nearly all the space of the channels is accessible.

We analyzed the binding manner and energy in the $\mathrm{V}$ corner confinement. Each NR molecule receives 9 close $\mathrm{C}^{-}$ $\mathrm{H} \cdots \pi$ interactions $(<3.0 \AA)^{[11]}$ with the $\mathrm{C}-\mathrm{H}$ units as well as attractive London dispersion forces (see Figure S10) from both walls. The multipoint interactions over 5 layers of a $1.8 \mathrm{~nm}$ long channel (Figure $4 \mathrm{c}$ ) lead to a gain in binding energy of $41.1 \mathrm{kcal} \mathrm{mol}^{-1}$. We virtually inserted C6 into the channel and observed a binding energy of $40.6 \mathrm{kcal} \mathrm{mol}^{-1}$, which is of the same level as that of NR (see Table S4). This simulation excludes the possibility that C6 could once enter the channel.

We explored the possibility of size recognition in the NR/ C6 and NR/DAPC mixtures. The addition of HFPTP-BPDA$\mathrm{COF}$ to a NR/C6 mixture caused the disappearance of the NR absorbance (Figure 5a). After only 2 min, NR was completely sorted into the channels (Figure 5b, red circles), while C6 was left in solution (Figure $5 \mathrm{~b}$, black circle). The decrement at $446 \mathrm{~nm}$ is associated with a certain absorbance of NR at $446 \mathrm{~nm}$. Indeed, after careful elimination of the effect of NR according to the Beer-Lambert law, the absorbance of the C6 molecules was retained. Clearly, the quickness of NR uptake is not interfered with by the existence of the same number of a)
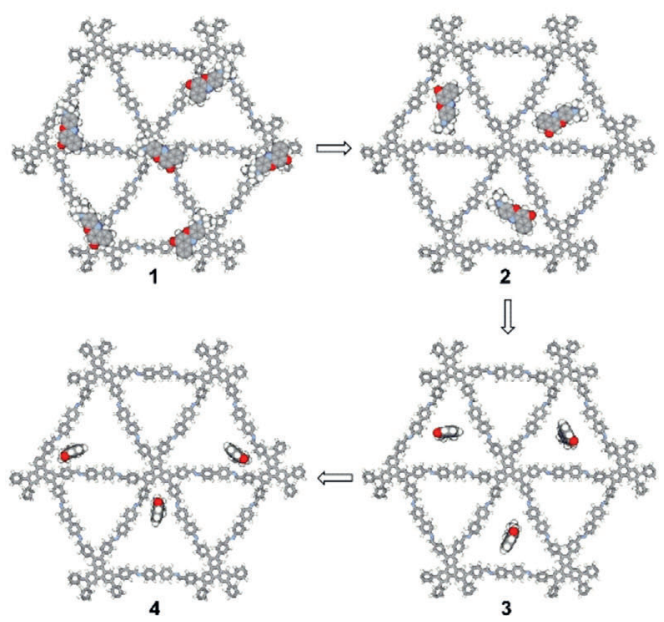

b)

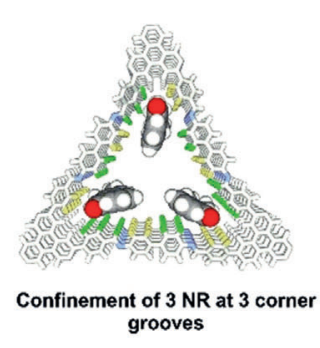

d)
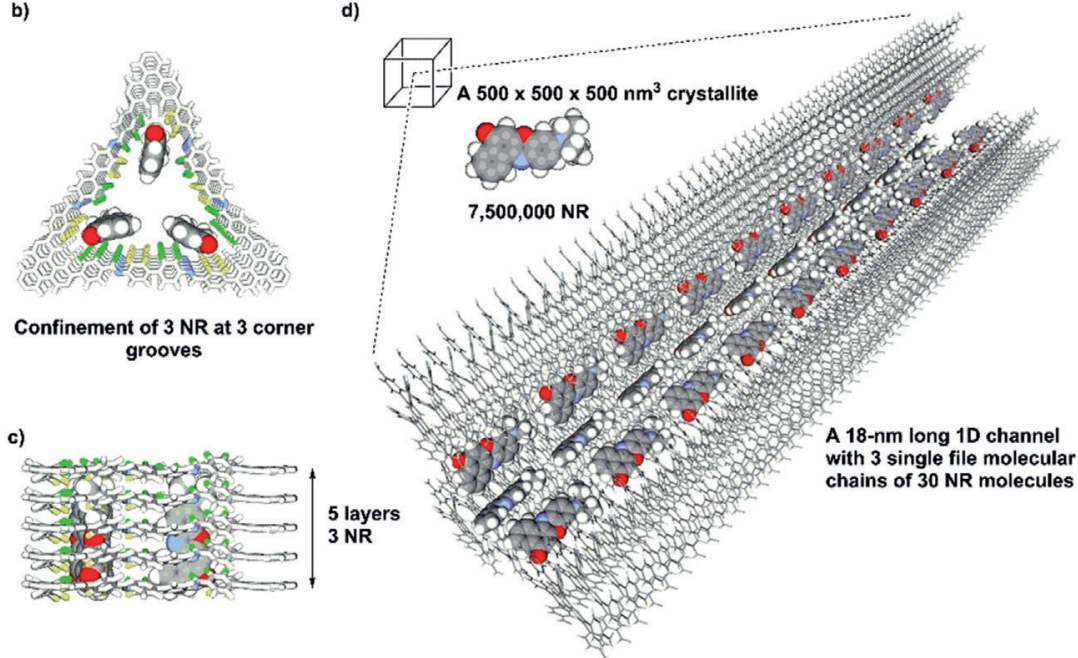

Figure 4. a) Process of size recognition and nanoconfinement by HFPTP-BPDA-COF. b) Top and c) side views of the reconstructed structure of three NR molecules in a $15.5 \AA$ triangular channel over five layers and a length of $1.8 \mathrm{~nm}$. d) A cubic HFPTP-BPDA-COF crystallite with the dimensions of only $500 \times 500 \times 500 \mathrm{~nm}^{3}$ confines over $7500000 \mathrm{NR}$ molecules. Inset: Reconstructed structure of a triangular channel in 50 layers over a length of $18 \mathrm{~nm}$ for the confinement of $30 \mathrm{NR}$ molecules into three single-file molecular chains at its nanogrooves. 
a)
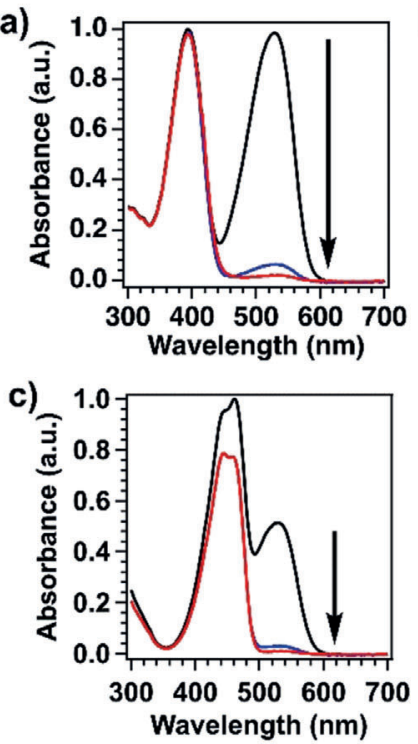

e)

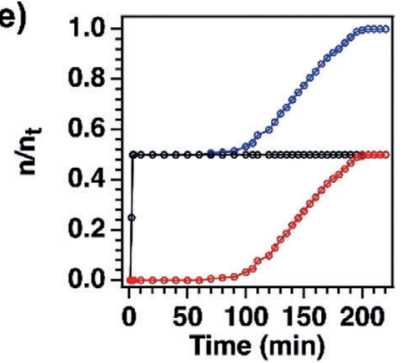

b)
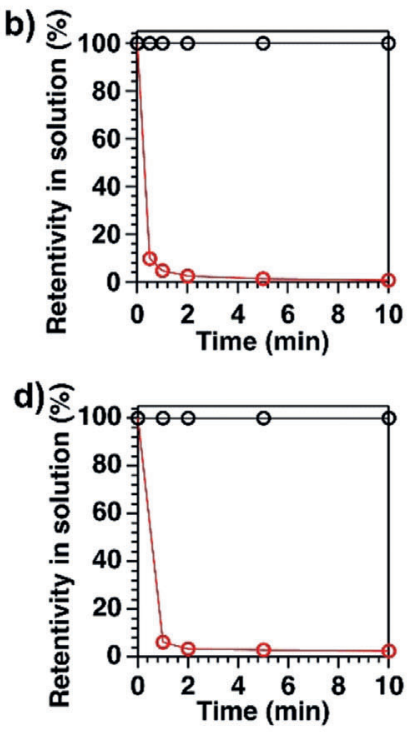

f)

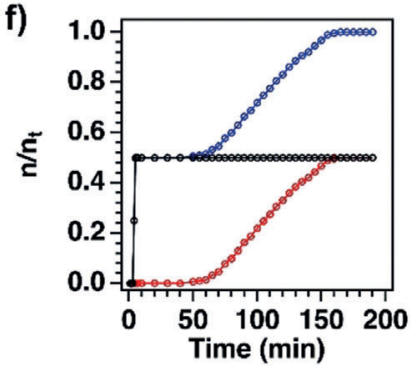

Figure 5. a) Electronic absorption spectral change of a mixture of NR and DAPC (1:1 molar ratio) in THF upon the addition of HTP-BDACOF (black curve, pristine; blue curve, after $30 \mathrm{~s}$; red curve, after 2 min). b) Time-dependent retentivity of NR (red circles and curve) and DAPC (black circles and curve) in solution. NR is sorted into the channels and DAPC is retained in solution. c) Electronic absorption spectral change of a mixture of NR and C6 (1:1 molar ratio) in THF upon the addition of HTP-BDA-COF (black curve, pristine; blue curve, after $30 \mathrm{~s}$; red curve, after $2 \mathrm{~min}$ ). d) Time-dependent retentivity of NR (red circles and curve) and C6 (black circles and curve) in solution. NR is sorted into the channels and C6 is retained in solution. e) COF column flow-out charts of C6 (black curve), NR (red curve), and C6 plus NR (blue curve) for a NR/C6 mixture (1:1 molar ratio) at ambient temperature and pressure. f) COF column flow-out charts of DAPC (black curve), NR (red curve), and DAPC plus NR (blue curve) for a NR/DAPC mixture (1:1 molar ratio) at ambient temperature and pressure.

C6 molecules. For the NR/DAPC mixture, an instant drop in the NR absorption band (Figure 5c) confirmed that the 1D channels sort NR molecules quickly into the pores upon only a short exposure (Figure $5 \mathrm{~d}$, red circles) and leave DAPC molecules in the solution phase (Figure $5 \mathrm{~d}$, black circles). Thus, the triangular channels can recognize size differences in mixtures and endow HFPTP-BPDA-COF with the potential of exceptional separation. As COFs are versatile in pore-wall engineering, ${ }^{[12]}$ our strategy offers an approach to designable 1D channels for precise size recognition, selective transport, and quick separation.

We demonstrated the separation of NR/C6 and NR/ DAPC mixtures using HFPTP-BPDA-COF columns. A THF

solution of NR/C6 $\left(0.02 \mathrm{mmol}\right.$ in total, $\left.1 / 1 \mathrm{~mol} \mathrm{~mol}^{-1}\right)$ was subjected to an open column of HFPTP-BPDA-COF (30 mg; $1.5 \mathrm{~cm}$ long in column) followed by a THF flow at ambient temperature and pressure. The green-colored C6 fraction came out of the column as soon as the THF solvent flowed out, thus enabling swift separation of C6 from NR. Indeed, the C6 fraction flushed out completely within 2 min to yield $100 \%$ purity (Figure 5e, black curve; see also Figure S11), thus leading to separation with infinite selectivity (Figure $5 \mathrm{e}$, blue curve). The NR fraction was also pure and came out over a time range of 70-200 min as the NR molecules were sorted into the channels (Figure 5e, red curve). In the same way, the HFPTP-BPDA-COF column separated the NR/DAPC mixture (Figure $5 \mathrm{f}$, blue curve) into DAPC within 2 min (Figure $5 \mathrm{f}$, black curve; see also Figure S12) and NR in 60$160 \mathrm{~min}$ (Figure $5 \mathrm{f}$, red curve). These results also suggest that the triangular channels enable quick uptake but slow release; this character is helpful for a molecular delivery system to achieve a constant release. ${ }^{[13]}$ Notably, the COF columns retained their functions (see Figure S13) as well as crystallinity and porosity (see Figures S14 and S15) over five cycles. Finally, we used commercial silica gel $(700 \mathrm{mg}$; 23 -fold the amount of $\mathrm{COF}$ by weight; $7 \mathrm{~cm}$ long column; THF) to separate the mixtures by their polarity differences in THF and observed that they could not be separated (see Figure S16). Thus, the recognition and separation as well as transportation by the $1 \mathrm{D}$ channels are not based on polarity but driven solely by subtle differences in molecular size.

\section{Conclusion}

In summary, we have established a strategy for size recognition of synthetic 1D nanochannels, by exploring a latticed framework with shape-persistent triangular pores. Importantly, we found that the $2 \mathrm{D} \pi$ surface of the framework, the triangular aperture, and the pore walls with built-in $\mathrm{C}-\mathrm{H}$ sequences are key structural parameters in size recognition. Their cooperativeness renders the $\mathrm{COF}$ able to combine precise size recognition of one atom difference, selective docking and transport, and instant separation in one material, thus demonstrating the strength of 1D channels for accurate molecular recognition and separation-an important goal for the implementation of porous materials. These unique properties of synthetic 1D channels have been established for the first time in a COF and could exert great impact on the further advance of the field. We envisage that synthetic $1 \mathrm{D}$ channels could offer a promising platform for designing robust size-recognition devices that are useful in adsorption, transportation, and separation.

\section{Acknowledgements}

D.J. acknowledges an MOE Tier 1 grant (R-143-000-A71-114) and NUS startup grant (R-143-000-A28-133). M.A. acknowledges support from the EPSRC (EP/S015868/1) and HPC resources of T.H. through the Materials Chemistry Consortium (EP/P020194). T.H. acknowledges financial support by 
the Deutsche Forschungsgemeinschaft, SPP 1928 COORNETs, under contract number HE 3543/30-1.

\section{Conflict of interest}

The authors declare no conflict of interest.

Keywords: covalent organic frameworks . molecular recognition - nanochannels - nanostructures . separation

[1] a) D. A. Doyle, J. M. Cabral, R. A. Pfuetzner, A. Kuo, J. M. Gulbis, S. L. Cohen, B. T. Chait, R. MacKinnon, Science 1998, 280, 69-77; b) N. Huang, P. Wang, D. Jiang, Nat. Rev. Mater. 2016, 1,16068 .

[2] V. Abetz, P. F. W. Simon, Polymer Science, Vol. 189 (Ed.: V. Abetz), Springer, Berlin, 2005, pp. 125-212.

[3] C. T. Kresge, M. E. Leonowicz, W. J. Roth, J. C. Vartuli, J. S. Beck, Nature 1992, 359, 710-712.

[4] a) P. J. Waller, F. Gándara, O. M. Yaghi, Acc. Chem. Res. 2015 48, 3053-3063; b) J. W. Colson, A. R. Woll, A. Mukherjee, M. P. Levendorf, E. L. Spitler, V. B. Shields, M. G. Spencer, J. Park, W. R. Dichtel, Science 2011, 332, 228-231; c) J. Guo et al., Nat. Commun. 2013, 4, 2736.
[5] a) T. Ma et al., Science 2018, 361, 48-52; b) X. Guan, H. Li, Y. Ma, M. Xue, Q. Fang, Y. Yan, V. Valtchev, S. Qiu, Nat. Chem. 2019, 11, 587-594; c) L. Ascherl et al., Nat. Chem. 2016, 8, 310 316; d) S. Kandambeth, A. Mallick, B. Lukose, M. V. Mane, T. Heine, R. Banerjee, J. Am. Chem. Soc. 2012, 134, 19524-19527; e) M. R. Rao, Y. Fang, S. De Feyter, D. F. Perepichka, J. Am. Chem. Soc. 2017, 139, 2421-2427; f) R.-B. Lin, B. Chen, Joule 2018, 2, 1030-1032; g) E. Jin, J. Li, K. Geng, Q. Jiang, H. Xu, Q. Xu, D. Jiang, Nat. Commun. 2018, 9, 4143; h) L. Zou, X. Yang, S. Yuan, H.-C. Zhou, CrystEngComm 2017, 19, 4868-4871.

[6] P. Wang, Q. Xu, Z. Li, W. Jiang, Q. Jiang, D. Jiang, Adv. Mater. 2018, 30, 1801991.

[7] a) B. Aradi, B. Hourahine, T. Frauenheim, J. Phys. Chem. A 2007, 111, 5678-5684; b) http://www.dftb.org.

[8] S. Feng, H. Xu, C. Zhang, Y. Chen, J. Zeng, D. Jiang, J.-X. Jiang, Chem. Commun. 2017, 53, 11334-11337.

[9] M. A. Addicoat, S. Fukuoka, A. J. Page, S. Irle, J. Comput. Chem. 2013, 34, 2591-2600.

[10] G. Das, T. Skorjanc, T. Prakasam, S. Nuryyeva, J.-C. Olsen, A. Trabolsi, RSC Adv. 2017, 7, 3594-3598.

[11] M. Nishio, Y. Umezawa, M. Hirota, Y. Takeuchi, Tetrahedron 1995, $51,8665-8701$.

[12] a) A. Nagai, Z. Guo, X. Feng, S. Jin, X. Chen, X. Ding, D. Jiang, Nat. Commun. 2011, 2, 536; b) N. Huang, R. Krishna, D. Jiang, J. Am. Chem. Soc. 2015, 137, 7079-7082.

[13] K. Park, J. Controlled Release 2014, 190, 3-8.

Manuscript received: August 4, 2019

Accepted manuscript online: August 15, 2019

Version of record online: $\square \square$ u, पuा 


\section{Research Articles}

\section{Covalent Organic Frameworks}

P. Wang, X. Chen, Q. Jiang, M. Addicoat,

N. Huang, S. Dalapati, T. Heine, F. Huo,

D. Jiang*

IIII-IIII

High-Precision Size Recognition and Separation in Synthetic 1D Nanochannels
The right channel: Open 1D channels were able to recognize differences in molecular size when the pores were designed with a triangular shape and discrete size in covalent organic frameworks. This finding introduces the possibility of using 1D channels for selective transport and instant molecular separation with infinite selectivity (see picture). 